TTK-12-19

\title{
Top quark and leptonic charge asymmetries for the Tevatron and LHC
}

\author{
Werner Bernreuther ${ }^{*}$ and Zong-Guo Si \\ ${ }^{a}$ Institut für Theoretische Physik, RWTH Aachen University, 52056 Aachen, \\ Germany \\ ${ }^{b}$ Department of Physics, Shandong University, Jinan, Shandong 250100, \\ China
}

\begin{abstract}
We compute, for $t \bar{t}$ production at the LHC and at the Tevatron, several charge asymmetries to next-to-leading oder (NLO) QCD, including also the electromagnetic and weak-interaction corrections. We calculate these asymmetries both inclusively and with additional kinematic cuts and compare our results, where possible, with recent experimental results and with Standard Model (SM) predictions. The $t \bar{t}$ asymmetries induce also corresponding asymmetries for the charged leptons from semileptonic top-quark decay. Although these asymmetries are, in the SM, smaller than the corresponding ones for top quarks, they are expected to be measurable quite precisely. In fact, measurement of a lepton asymmetry in $\ell+$ jets events was reported by the $\mathrm{D} \emptyset[2$ and CDF [7] experiments. We analyze and compute to NLO in the gauge couplings leptonic charge asymmetries for dileptonic and semileptonic $t \bar{t}$ events, with and without acceptance cuts, at the Tevatron and the LHC.
\end{abstract}

PACS number(s): 12.38.Bx, 13.88.+e, 14.65.Ha

Keywords: hadron collider physics, top quarks, charge asymmetry, forward-backward asymmetry

\footnotetext{
*breuther@physik.rwth-aachen.de

†zgsi@sdu.edu.cn
} 


\section{Introduction}

So far, almost all of the experimental results from the Tevatron and the LHC on top quark production and decay imply that this quark behaves pretty much as expected from the Standard Model - the exception being the measurements of the charge asymmetry in $t \bar{t}$ production at the Tevatron by the CDF and $\mathrm{D} \emptyset$ experiments reported in [1, 2], which are (considerably) higher than the SM predictions [3 6]. In particular, a 3.4 $\sigma$ deviation from the SM was cited in [1] for the CDF determination [1] of the $t \bar{t}$ rest frame asymmetry for high pair-invariant mass, $A^{t \bar{t}}\left(M_{t \bar{t}}>450 \mathrm{GeV}\right)$. This triggered a very large number of investigations on possible new physics contributions to $t \bar{t}$ production. (For recent reviews, see for instance [8 10.) A recent CDF measurement 7] of this observable based on a larger data sample and, on the theory side, the incorporation of the complete $\mathcal{O}\left(\alpha_{s}^{2} \alpha\right)$ electroweak corrections [11] alleviated this tension as far as $A^{t \bar{t}}\left(M_{t \bar{t}}>450 \mathrm{GeV}\right)$ is concerned, but did not remove it.

The situation is unclear for several reasons. The $\mathrm{D} \emptyset$ experiment at the Tevatron did not confirm [2] the significant enhancement of the $t \bar{t}$ rest-frame asymmetry at high $M_{t \bar{t}}$ seen by CDF. On the other hand, D $\emptyset$ measured a leptonic charge asymmetry $A^{\ell}$ in $t \bar{t} \rightarrow \ell+$ jets [2], which is considerably larger than the corresponding SM prediction [12], while a recent CDF measurement [7], which is however not yet corrected for detector effects and acceptance, agrees with it. Moreover, the $t \bar{t}$ charge asymmetries $A_{C}$ measured by the CMS [13, 14] and ATLAS [15] collaborations at the LHC agree, within the present uncertainties, with the SM calculations [16]. Obviously, it is of prime importance to explore and hopefully clarify this topic in detail in the (near) future, both by experiment and theory.

As the SM-induced charge asymmetry in $t \bar{t}$ production at the LHC is small, a number of observables related to $A_{C}$ have been proposed and analyzed, including those in [6, 16, 23], that enhance the (predominantly QCD induced) effect and serve to discriminate between the $\mathrm{SM}$ and various new physics models. In this paper we compute some of these asymmetries at next-to-leading order (NLO) in the SM gauge couplings. Here, this notation refers to the computation of the numerators of the asymmetries to order $\alpha_{s}^{3}$ in the QCD coupling including the mixed QCD-QED and mixed QCD-weak interaction corrections. For some of these observables, respective results were recently obtained in the literature [11,16-18] with which we compare; some of our results are new. So far, most of the predictions were made at the $t \bar{t}$ production level, while the experimental measurements of the charge asymmetries at the "reconstruction level" were unfolded, i.e., corrected for detector acceptance and resolution, to obtain the corresponding $t \bar{t}$ "production level" asymmetries.

The $t \bar{t}$ asymmetries induce also corresponding asymmetries for the charged leptons from semileptonic top-quark decay. Although these asymmetries are, in the SM, smaller than the corresponding ones for top quarks [12], they should be measurable more precisely. In fact, measurement of a lepton asymmetry in $\ell+$ jets events was reported by D $\emptyset[2]$ and CDF [7]. We analyze and compute to NLO in the gauge couplings leptonic charge asymmetries for dileptonic and semileptonic $t \bar{t}$ events, with and without acceptance cuts, at the Tevatron and the LHC. This extends our previous results for the Tevatron 12 .

It seems appropriate to briefly recapitulate here the status of the SM predictions concerning the $t \bar{t}$ charge asymmetries in hadronic production. In the SM, the leading-order effect is induced by the NLO QCD, i.e., the $\mathcal{O}\left(\alpha_{s}^{3}\right)$ contributions $d \sigma_{A, t \bar{t}}$ to the differential $t \bar{t}$ cross

\footnotetext{
${ }^{1}$ An interesting proposal of "collider independent" charge asymmetries was recently made in [24].
} 
section which are odd with respect to the exchange of $t \leftrightarrow \bar{t}$. The first dedicated NLO QCD prediction of the charge asymmetry, including an estimate of the electroweak contributions, was made in [3, 4]. Subsequent analyses were done in [5, 6]. In [12] the mixed QCD-weak corrections of $\mathcal{O}\left(\alpha_{s}^{2} \alpha\right)$ were included. Ref. [11] determined, besides the weak contributions, also the QCD-QED contributions of $\mathcal{O}\left(\alpha_{s}^{2} \alpha\right)$ which are, in fact, more important than the weakinteraction corrections, and obtained predictions of the Tevatron asymmetries to NLO in the SM gauge couplings; cf. also [16]. The NNLO QCD corrections to $d \sigma_{A, t \bar{t}}$ are not yet known 2 . The fixed-order NLO QCD computations were supplemented by soft-gluon resummation at next-to-leading (NLL) [31] and next-to-next-to-leading (NNLL) 32 34 logarithmic order. These corrections do not alter the fixed-order NLO QCD results significantly. The QCDinduced charge asymmetries can and are being computed also with the widely used NLO QCD Monte Carlo programs [35-39]. An issue, which in the past has been a source of confusion between theorists and experimentalists, is how the asymmetries are computed in the context of NLO Monte Carlo simulations, see Sect. 2.

The paper is organized as follows. In Sect. 2 we compute a number of $t \bar{t}$ charge asymmetries at NLO in the SM gauge couplings for the Tevatron and the LHC at 7, 8, and $14 \mathrm{TeV}$ center-of-mass energy and compare, where possible, with experimental results and other SM calculations. In Sect. 3 we make corresponding SM predictions for two leptonic asymmetries for dileptonic and lepton plus jets $t \bar{t}$ events at the Tevatron and for some leptonic asymmetries for dileptonic final states at the LHC. Sect. 4 contains a summary and outlook.

\section{Top-quark charge and forward-backward asymmetries}

In this section we consider various forward-backward and charge asymmetries for the Tevatron and LHC at the level of $t \bar{t}$ on-shell intermediate states and calculate these asymmetries within the SM.

\subsection{Tevatron}

First we compute the top-quark laboratory- and rest-frame charge/forward-backward asymmetries for the Tevatron to NLO QCD including the photonic and weak-interaction contributions. The sole purpose of this section is to compare the results of our computational setup with previous SM computations of these asymmetries [3, 6, 11, 12, 16, 31, 35], and also with recent experimental results [1,2,7].

For top-quark pair production at the Tevatron, $p \bar{p} \rightarrow t \bar{t}+X$, the differential and integrated charge asymmetry, $A(y)$ and $A$, are defined by

$$
A(y)=\frac{N\left(y_{t}\right)-N\left(y_{\bar{t}}\right)}{N\left(y_{t}\right)+N\left(y_{\bar{t}}\right)}, \quad A=\frac{N\left(y_{t}>0\right)-N\left(y_{\bar{t}}>0\right)}{N\left(y_{t}>0\right)+N\left(y_{\bar{t}}>0\right)},
$$

where $y_{t}, y_{\bar{t}}$ denote the rapidities of the $t$ and $\bar{t}$ quark in the laboratory frame, and $N(y)=$ $d \sigma_{t \bar{t}} / d y$. CP invariance implies that for $t \bar{t}$ production at the Tevatron $N\left(y_{\bar{t}}\right)=N\left(-y_{t}\right)$,

\footnotetext{
${ }^{2}$ For $t \bar{t}+$ jet events, QCD induces a charge asymmetry already at tree-level, which receives large corrections at NLO [26 30]. Ref. 28] argues that the inclusive $t \bar{t}$ asymmetries may not receive such large QCD corrections beyond NLO QCD.
} 
which in turn implies that $A$ is equal to the forward-backward asymmetry of the top quark:

$$
A_{F B}^{t}=\frac{N\left(y_{t}>0\right)-N\left(y_{t}<0\right)}{N\left(y_{t}>0\right)+N\left(y_{t}<0\right)} \quad \text { and } \quad A_{F B}^{\bar{t}}=-A_{F B}^{t} .
$$

Another important observable is the pair asymmetry or $t \bar{t}$ rest-frame asymmetry

$$
A^{t \bar{t}}=\frac{N(\Delta y>0)-N(\Delta y<0)}{N(\Delta y>0)+N(\Delta y<0)},
$$

where $\Delta y=y_{t}-y_{\bar{t}}$. As $\Delta y$ is boost-invariant along the beam axis, this rapidity difference is, in the limit of small $p_{T}$ of the $t \bar{t}$ system, the same in the hadronic and the $t \bar{t}$ rest frame. The sign of $\Delta y$ is, in fact, invariant under such a boost. The asymmetry (3) is, for kinematical reasons, larger than (2).

The Bose symmetry of the $g g$ state precludes a contribution to the asymmetries $A, A_{F B}^{t}$, and $A^{t \bar{t}}$ from $g g \rightarrow t \bar{t} X$ - irrespective of whether or not the production density matrix $R_{g g}$ contains $\mathrm{P}$ - and/or CP-violating pieces. The asymmetries are generated by the interference of even and odd terms under $t \leftrightarrow \bar{t}$ - while the initial partons are kept fixed - in the amplitudes for $q \bar{q} \rightarrow t \bar{t} X$ and, likewise, for $g q \rightarrow t \bar{t} q(+X)$ and $g \bar{q} \rightarrow t \bar{t} \bar{q}(+X)$.

In the SM the dominant contributions to (11) - (3) arise from the NLO QCD corrections to $t \bar{t}$ production by $q \bar{q}$ annihilation, i.e., terms of order $\alpha_{s}^{3}$ in the partonic cross section $d \hat{\sigma}(q \bar{q} \rightarrow t \bar{t} X)$ which are antisymmetric under the interchange of $t$ and $\bar{t}$. These terms comprise, for $q \bar{q} \rightarrow t \bar{t}$, the antisymmetric part of the interference of the Born diagram with the 1-loop box and crossed box diagrams and, for $q \bar{q} \rightarrow t \bar{t} g$, the antisymmetric part of the interference of initial and final state radiation. In addition, antisymmetric interference terms of order $\alpha_{s}^{3}$ in the squared matrix elements of $g q \rightarrow t \bar{t} q$ and $g \bar{q} \rightarrow t \bar{t} \bar{q}$, respectively, contribute also to the above asymmetries. At the Tevatron, they are numerically irrelevant, while at the LHC they may reach a sizeable fraction of the contributions from $q \bar{q}$ annihilation (see below).

As was pointed out in [11, the mixed QCD-QED contributions of order $\alpha_{s}^{2} \alpha$ to the asymmetries from $q \bar{q} \rightarrow t \bar{t}, t \bar{t} g, t \bar{t} \gamma$ are important. (These corrections had been estimated previously in [4].) At the level of the $q \bar{q}$ initial states, the ratio of the mixed QCD-QED and pure QCD contributions is $R_{q}=(36 / 5) Q_{q} Q_{t} \alpha / \alpha_{s}$ [11] (where $Q_{a}$ denotes the charge of quark $a$ in units of $e$ ). For $p \bar{p}$ collisions at the Tevatron this implies that the ratio of the corresponding contributions to (1) - (3) is about $18 \%$. At the LHC this ratio decreases to about $13 \%$ because the ratio of $u \bar{u}$ and $d \bar{d}$ collisions decreases from about $4: 1$ at the Tevatron to $2: 1$ at the LHC.

The (nominally) leading effects of the weak interactions on the asymmetries are as follows. At Born level there is the contribution of $\mathcal{O}\left(\alpha^{2}\right)$ from the antisymmetric terms of the squared amplitudes of $q \bar{q} \rightarrow \gamma, Z \rightarrow t \bar{t}$. Then there are antisymmetric terms in the mixed QCD-weak corrections of $\mathcal{O}\left(\alpha_{s}^{2} \alpha\right)$ to $q \bar{q} \rightarrow t \bar{t}(g)$. These are contained in i) the interferences of the $\mathcal{O}\left(\alpha_{s}^{2}\right)$ two-gluon box diagrams with the Born $Z$-exchange diagram and of the $\mathcal{O}\left(\alpha_{s} \alpha\right)$ Z-gluon box diagrams with the Born gluon exchange diagram, and ii) in the interferences of the $\mathcal{O}\left(g_{s}^{3}\right)$ and $\mathcal{O}\left(g_{s} e^{2}\right)$ gluon bremsstrahlung diagrams. (The contribution from $Z$ boson radiation, $q \bar{q} \rightarrow t \bar{t} Z$, to the inclusive asymmetries is very small and will be neglected.) At the Tevatron and the LHC these weak interaction corrections increase the QCD asymmetries by a few 
percent (cf. [11, 12, 16] and below). The weak interactions induce also parity-violating form factors at 1-loop in the $q \bar{q} g$ and $t \bar{t} g$ vertices; however, they make, at $\mathcal{O}\left(\alpha_{s}^{2} \alpha\right)$, no contribution to the antisymmetric part of $d \hat{\sigma}(q \bar{q} \rightarrow t \bar{t} X)$. There are also mixed QCD-weak contributions of $\mathcal{O}\left(\alpha_{s}^{2} \alpha\right)$ and $\mathcal{O}\left(\alpha_{s} \alpha^{2}\right)$ to the asymmetries from $g q(\bar{q}) \rightarrow t \bar{t} q(\bar{q})$. They are negligibly small for the Tevatron, but at the LHC they are of comparable size as the mixed QCD-weak contributions to $q \bar{q}$ annihilation (see Sect. 2.2).

In the following we compute the asymmetries (2) and (3), taking into account in the numerators the $\mathcal{O}\left(\alpha_{s}^{3}\right)$ QCD and the $\mathcal{O}\left(\alpha^{2}\right)$ and $\mathcal{O}\left(\alpha_{s}^{2} \alpha\right)$ electroweak corrections as discussed above. (As to the weak interaction corrections, we use our previous results [40 42], cf. also [43 45].) To this order, a consistent fixed-order perturbative expansion of the ratios (2), (3) precludes taking into account the NLO QCD corrections to the denominators. Therefore we evaluate the denominators of all the asymmetries considered in this paper with LO QCD matrix elements (as was done in [3, 4, 6, 11, 12, 16]). As to the use of parton distribution functions (PDF), we evaluate both the numerator and the denominator of the asymmetries with NLO $\mathrm{PDF} 3$.

We use $m_{t}=173.1 \mathrm{GeV}$ (on-shell mass), the QED coupling $\alpha\left(m_{Z}\right)=0.008$, and the weak mixing angle $\sin ^{2} \theta_{W}=0.23$. We use the CTEQ6.6M PDF [47] and the respective value of $\alpha_{s}\left(m_{Z}\right)$ provided by this set. The same value $\mu$ is used for the renormalization and the factorization scale, and numerical results are given for $\mu=m_{t} / 2, m_{t}$, and $2 m_{t}$. These scale choices are purely conventional. The variation of the asymmetries within this range of $\mu$ are no substitute for a realistic assessment of the theory uncertainites; see the corresponding remarks on page 7 below.

In Tables 1 and 2 we present our results for the laboratory-frame and $t \bar{t}$ rest-frame asymmetry (2) and (3) , respectively. In the first rows, the QCD and electroweak contributions to the numerators of these asymmetries are given. (Notice that contributions from quark flavors $q \neq u, d$ are, after convolution with the PDF, symmetric under interchange of $t$ and $\bar{t}$ and therefore do not contribute to the numerators.) In the row labeled $q g$ the sum of the contributions from the $q g$ and $\bar{q} g$ fusion processes is given - for the sole purpose of showing that it can be safely neglected for the Tevatron, which will be done in the following. The tables show what has already been mentioned above: the mixed QCD-QED and QCD-weak contributions increase the QCD asymmetries at the Tevatron by $18 \%$ and $5 \%$, respectively, i.e. in total by $23 \%$.

In Table 3 we collect our results for $A_{F B}^{t}$ and for $A^{t \bar{t}}$ without and with cuts on $|\Delta y|$ and $M_{t \bar{t}}$ and list, for comparison, also results from the CDF and D $\emptyset$ experiments [1, 2, 7]. The experimental results for the asymmetries are the unfolded values at the $t \bar{t}$ production level, i.e., corrected for detector resolution and acceptance. The CDF results given in column 3 of this data are from lepton plus jets events based on an integrated luminosity of $8.7 \mathrm{fb}^{-1}$, while column 2 resulted from the analysis of $L_{i n t}=5.3 \mathrm{fb}^{-1}$ [1]. The $\mathrm{D} \emptyset$ collaboration [2] did not find statistically sensitive dependencies of $A^{t \bar{t}}$ on $|\Delta y|$ and $M_{t \bar{t}}$ [2] and therefore did not publish unfolded numbers for the observables listed in rows 2 - 5 of Table 3 .

\footnotetext{
${ }^{3}$ In [12] a different procedure was used. The numerators were evaluated with NLO PDF while in the denominators LO PDF and the same value of $\alpha_{s}$ as in the numerator were used. This yields slightly larger asymmetries (by $\sim 4 \%$ ) than those given below.
} 


\begin{tabular}{|c|c|c|c|c|}
\hline \multicolumn{2}{|c|}{$N_{F B}^{t}(\mathrm{pb})$} & $\mu=m_{t} / 2$ & $\mu=m_{t}$ & $\mu=2 m_{t}$ \\
\hline$O\left(\alpha_{s}^{3}\right)$ & $u \bar{u}$ & 0.3328 & 0.2183 & 0.1489 \\
\cline { 3 - 5 } & $d \bar{d}$ & 0.0591 & 0.0381 & 0.0257 \\
\cline { 3 - 5 } & $q g$ & $4.1 \times 10^{-5}$ & $2.6 \times 10^{-5}$ & $1.7 \times 10^{-5}$ \\
\hline$O\left(\alpha^{2}\right)$ & $u \bar{u}$ & $9.4 \times 10^{-3}$ & $8.3 \times 10^{-3}$ & $7.4 \times 10^{-3}$ \\
\cline { 3 - 5 } & $d \bar{d}$ & $1.2 \times 10^{-3}$ & $1.1 \times 10^{-3}$ & $9.2 \times 10^{-4}$ \\
\hline$O\left(\alpha \alpha_{s}^{2}\right)_{\text {weak }}$ & $u \bar{u}$ & $7.1 \times 10^{-3}$ & $5.2 \times 10^{-3}$ & $3.8 \times 10^{-3}$ \\
\cline { 3 - 5 } & $d \bar{d}$ & $-2.2 \times 10^{-3}$ & $-1.6 \times 10^{-3}$ & $-1.2 \times 10^{-3}$ \\
\hline$O\left(\alpha \alpha_{s}^{2}\right)_{Q E D}$ & $u \bar{u}$ & 0.0692 & 0.0502 & 0.0375 \\
\cline { 3 - 5 } & $d \bar{d}$ & $-6.1 \times 10^{-3}$ & $-4.4 \times 10^{-3}$ & $-3.2 \times 10^{-3}$ \\
\hline \multicolumn{2}{|c|}{ total } & 0.4701 & 0.3151 & 0.2198 \\
\hline \hline$\sigma_{Q C D}^{L O}(\mathrm{pb})$ & 7.618 & 5.456 & 4.030 \\
\hline \hline$A_{F B}^{t}(\%)$ & 6.17 & 5.77 & 5.46 \\
\hline
\end{tabular}

Table 1: The contributions to the numerator of the $t$-quark forward-backward laboratoryframe asymmetry (2) at the Tevatron for three different scales. The denominator of (2), $\sigma_{Q C D}^{L O}=\sigma_{t \bar{t}}$, is computed at leading-order QCD.

\begin{tabular}{|c|c|c|c|c|}
\hline \multicolumn{2}{|c|}{$N^{t t}(\mathrm{pb})$} & $\mu=m_{t} / 2$ & $\mu=m_{t}$ & $\mu=2 m_{t}$ \\
\hline$O\left(\alpha_{s}^{3}\right)$ & $u \bar{u}$ & 0.5014 & 0.3297 & 0.2251 \\
\cline { 3 - 5 } & $d \bar{d}$ & 0.0899 & 0.0582 & 0.0392 \\
\cline { 3 - 5 } & $q g$ & $7.6 \times 10^{-5}$ & $3.4 \times 10^{-5}$ & $2.9 \times 10^{-5}$ \\
\hline$O\left(\alpha^{2}\right)$ & $u \bar{u}$ & $1.47 \times 10^{-2}$ & $1.29 \times 10^{-2}$ & $1.15 \times 10^{-2}$ \\
\cline { 3 - 5 } & $d \bar{d}$ & $1.9 \times 10^{-3}$ & $1.6 \times 10^{-3}$ & $1.5 \times 10^{-3}$ \\
\hline$O\left(\alpha \alpha_{s}^{2}\right)_{\text {weak }}$ & $u \bar{u}$ & $10.7 \times 10^{-3}$ & $7.8 \times 10^{-3}$ & $5.8 \times 10^{-3}$ \\
\cline { 3 - 5 } & $d \bar{d}$ & $-3.4 \times 10^{-3}$ & $-2.4 \times 10^{-3}$ & $-1.8 \times 10^{-3}$ \\
\hline$O\left(\alpha \alpha_{s}^{2}\right)_{Q E D}$ & $u \bar{u}$ & 0.1047 & 0.0761 & 0.0569 \\
\cline { 3 - 5 } & $d \bar{d}$ & $-9.4 \times 10^{-3}$ & $-6.7 \times 10^{-3}$ & $-4.9 \times 10^{-3}$ \\
\hline total & & 0.7104 & 0.4772 & 0.3332 \\
\hline \hline$\sigma_{Q C D}^{L O}(\mathrm{pb})$ & 7.618 & 5.456 & 4.030 \\
\hline \hline$A^{t t}(\%)$ & 9.33 & 8.75 & 8.27 \\
\hline
\end{tabular}

Table 2: The contributions to the numerator of the $t \bar{t}$ rest-frame asymmetry (3) at the Tevatron for three different scales. 


\begin{tabular}{|c|c|c|c|c|}
\hline & CDF [1] & CDF [7] & D $\emptyset[2]$ & SM (this work) \\
\hline$A_{F B}^{t}$ & $0.150 \pm 0.055$ & & & $0.058 \pm 0.004$ \\
$A^{t \bar{t}}$ & $0.158 \pm 0.075$ & $0.162 \pm 0.047$ & $0.196 \pm 0.065$ & $0.088 \pm 0.006$ \\
$A^{t \bar{t}}(|\Delta y| \leq 1)$ & $0.026 \pm 0.118$ & $0.088 \pm 0.047$ & & $0.061_{-0.003}^{+0.004}$ \\
$A^{t \bar{t}}(|\Delta y|>1)$ & $0.611 \pm 0.256$ & $0.433 \pm 0.109$ & & $0.206_{-0.011}^{+0.011}$ \\
$A_{t \bar{t}}\left(M_{t \bar{t}} \leq 450 \mathrm{GeV}\right)$ & $-0.116 \pm 0.153$ & $0.078 \pm 0.054$ & & $0.062_{-0.003}^{+0.0003}$ \\
$A^{t \bar{t}}\left(M_{t \bar{t}}>450 \mathrm{GeV}\right)$ & $0.475 \pm 0.114$ & $0.296 \pm 0.067$ & & $0.129_{-0.006}^{+0.008}$ \\
\hline
\end{tabular}

Table 3: Unfolded experimental results from CDF [1,7] and D $\emptyset$ [2] for the laboratory- and $t \bar{t}$ rest-frame asymmetry at the Tevatron without and with cuts on $|\Delta y|$ and $M_{t \bar{t}}$ and our SM predictions (errors are scale-uncertainties only).

The $\mathrm{D} \emptyset$ and recent CDF results [7] on the inclusive rest-frame asymmetry $A^{t \bar{t}}$ are within $\sim 1.5 \sigma$ of our SM prediction. As to the rest-frame asymmetry with cuts: For $A^{t \bar{t}}(|\Delta y| \leq 1)$ and $A^{t \bar{t}}\left(M_{t \bar{t}} \leq 450 \mathrm{GeV}\right)$, and the recent CDF [7] and our SM results agree well, while the recent CDF determinations $A^{t \bar{t}}(|\Delta y|>1)$ and of $A^{t \bar{t}}\left(M_{t \bar{t}}>450 \mathrm{GeV}\right)$ deviates from our SM predictions by $\sim 2 \sigma$ and $\sim 2.4 \sigma$, respectively.

The asymmetry $A^{t \bar{t}}$ increases approximately linearly with $|\Delta y|$ and $M_{t \bar{t}}$. The slopes of these straight lines that were recently determined by the CDF experiment [7] are significantly larger than those obtained in the SM. A cut on the transverse momentum of the $t \bar{t}$ system has a significant effect on the size of the charge asymmetries. For instance, selecting $t \bar{t}$ events with low $p_{\perp}^{t \bar{t}}$ significantly increases the asymmetries [16]. This is due to the fact that the positive inclusive NLO QCD asymmetries are generated by the contribution from Born times virtual and soft gluon terms, which is positive and the contribution from hard gluon radiation, which is negative.

Experiments usually compare their results with predictions made with one of the widely used NLO QCD Monte-Carlo generators [36 39]. In these programs the electroweak contributions to the asymmetries are not included. More importantly, in these Monte-Carlo calculations the denominators of the asymmetries are determined with NLO QCD parton matrix elements, which reduces the asymmetry by up to $\sim 30 \%$ as compared to the procedure employed by us and in [11,16.

We now compare our results with other recent SM calculations of $A_{F B}^{t}$ and $A^{t \bar{t}}$. In [11] the various contributions to the asymmetries were also given in detail. Although we use a different PDF set than [11], our results of Tables 1, 2 and 3 agree well with the numbers of the corresponding Tables of that reference. Ref. [16] also used a PDF set different from ours and employed the strategy of evaluating the numerators and the denominators of the asymmetries with LO PDF. Moreover, the mixed QCD-weak corrections, which make only a small contribution, were taken into account only approximately in [16]. Our results agree also with those of [16]. The recent fixed-order NLO QCD computation of $A_{F B}^{t}$ of [35] uses NLO matrix elements in the denominator and therefore gets a smaller value than our pure QCD result 7.1(6)\% (cf. Table 2). Moreover, with this procedure the uncertainties due to scale variations become significantly larger than those given in Table 3, Ref. 34] computed the 
above asymmetries in pure QCD, at NLO plus next-to-next-to-leading logarithmic accuracy $(\mathrm{NLO}+\mathrm{NNLL})$, by performing corresponding resummations of logarithms due to soft and collinear gluons. One expects that these resummations (cf. also [31, 33]) provide more realistic estimates of the scale uncertainties than those resulting from the fixed order NLO predictions. The central values of $A_{F B}^{t}$ and $A^{t \bar{t}}$, without and with the above cuts on the latter asymmetry, given in [34] are essentially the same as those obtained at fixed order NLO QCD. This may not be surprising because soft and collinear radiation from top quarks (which is the physics behind taking into account threshold resummations) does not change the directions of $t$ and $\bar{t}$ and thus the asymmetries in an essential way.

In concluding this section we recall that the recent CDF determination of the high-mass asymmetry has reduced, but not erased the tension with the existing NLO SM predictions. We emphasize that the uncertainties due to scale variations of our SM results given in Tables [1, 2] and 3 underestimate the theory errors, which are, more realistically, of the order of $\sim 30 \%$. It remains to be seen whether a complete fixed-order NNLO QCD computation of the asymmetries will alleviate this tension.

\section{2. $\mathrm{LHC}$}

Let us first recall the salient features of the charge asymmetries in top-quark pair production in $p p$ collisions, $p p \rightarrow t \bar{t}+X$. At the LHC, the initial $p p$ state is an eigenstate of parity. Thus, $A_{F B}^{t}=A_{F B}^{\bar{t}}=0$ as long as only parity-invariant interactions are taken into account. In fact, the parity-violating terms of the weak corrections appear only in the $t$ - and/or $\bar{t}$-spin dependent terms of the inclusive partonic $t \bar{t}$ production density matrices and do, therefore, not contribute to the inclusive $t \bar{t}$ asymmetries when making predictions for top quarks summed over their spins. As a consequence, at the LHC the differential charge asymmetry $A(y)$ induced by the SM interactions must be symmetric with respect to $y=0$. However, QCD predicts that for large values of $|y|$ of the (anti)top rapidity, the $t \bar{t}$ sample is such that there are more $t$ than $\bar{t}$ quarks, while for small values of $|y|$ it is the other way around. Therefore, in the SM the differential charge asymmetry $A(y)>0$ in the forward and backward regions, while $A(y)<0$ in the central region. Thus one can define non-zero (integrated) asymmetries. The dominant contributions to the numerator of $A(y)$ are again due to the antisymmetric part $(t \leftrightarrow \bar{t})$ of the $q \bar{q}$ differential cross section. Contrary to the Tevatron, the antisymmetric contributions from $q g$ fusion are not negligibly small at the LHC (see below).

Because at the LHC the fraction of $t \bar{t}$ production by $q \bar{q}$ annihilation is significantly smaller than by $g g$ fusion, it is clear that the charge asymmetries are smaller than at the Tevatron. With suitable cuts one may enhance the asymmetries. For instance, in the SM one expects that the charge asymmetries increase in magnitude with $M_{t \bar{t}}$ because at the LHC the $q \bar{q}$ luminosity increases with respect to the $g g$ luminosity for increasing pair-invariant mass. Other ways to enhance the $q \bar{q}$-initiated fraction of $t \bar{t}$ and thus the ratio of the antisymmetric and symmetric part of the $t \bar{t}$ cross section is to select forward and/or backward events, to select $t \bar{t}$ events whose c.m. frame is highly boosted along the beam axis with respect to the laboratory frame, or to put a cut on the transverse momentum of the $t \bar{t}$ system. These observations have led to a number of suggestions for LHC observables [4, 6, 13, 15, 23] that

\footnotetext{
${ }^{4}$ According to [46] a judicious choice of scale-setting leads to a significant increase of the QCD-induced asymmetries.
} 
exhibit small, but non-zero SM-induced charge asymmetries and are useful in discriminating between various new physics models which were proposed to explain the Tevatron asymmetry. In the following analysis of various LHC charge asymmetries, we have taken into account in the computation of the respective numerators the $\mathcal{O}\left(\alpha_{s}^{3}\right)$ QCD and the $\mathcal{O}\left(\alpha^{2}\right)$ and $\mathcal{O}\left(\alpha_{s}^{2} \alpha\right)$ electroweak contributions as outlined in Sect. 2.1. As mentioned above, the antisymmetric contributions from $q g$ fusion of $O\left(\alpha_{s}^{3}\right)$ are not negligible at the LHC. For completeness, we take into account also the mixed QCD-QED corrections of $O\left(\alpha \alpha_{s}^{2}\right)$ to $q g$ fusion - see below. The denominators of the asymmetries are evaluated again with LO QCD matrix elements and the NLO PDF set CTEQ6.6M.

\section{Central and edge charge asymmetry}

Choosing a cut $y_{c}$ on the rapidities of the $t$ and $\bar{t}$ quarks, one may define central and edge (or forward) charge asymmetries $A_{C}, A_{E}$ [6, 18, 19]:

$$
\begin{aligned}
& A_{C}\left(y_{c}\right)=\frac{N\left(\left|y_{t}\right| \leq y_{c}\right)-N\left(\left|y_{\bar{t}}\right| \leq y_{c}\right)}{N\left(\left|y_{t}\right| \leq y_{c}\right)+N\left(\left|y_{\bar{t}}\right| \leq y_{c}\right)}, \\
& A_{E}\left(y_{c}\right)=\frac{N\left(y_{c} \leq\left|y_{t}\right|\right)-N\left(y_{c} \leq\left|y_{\bar{t}}\right|\right)}{N\left(y_{c} \leq\left|y_{t}\right|\right)+N\left(y_{c} \leq\left|y_{\bar{t}}\right|\right)},
\end{aligned}
$$

where the (anti)top rapidities are defined in the laboratory frame. The above discussion tells us that for suitably chosen $y_{c}$, the central asymmetry $A_{C}\left(y_{c}\right)<0$ and $A_{E}\left(y_{c}\right)>0$ in the SM. Because the fraction of $q \bar{q}$ initiated $t \bar{t}$ events, $\sigma_{q \bar{q} \rightarrow t \bar{t}} / \sigma_{t \bar{t}}$, is enhanced in the forward/backward region, $A_{E}$ will in general be larger than $\left|A_{C}\right|$. On the other hand, the event numbers decrease rapidly with increasing $|y|$; i.e., $y_{c}$ must be chosen appropriately for each of these observables in order to optimize the statistical sensitivity of $A_{E}$.

For the computation of the central asymmetry we choose $y_{c}=1$ and take into account $t \bar{t}$ events with $M_{t t} \geq M_{c}$. We choose $M_{c}=2 m_{t}, 0.5 \mathrm{TeV}, 0.7 \mathrm{TeV}$ and $1 \mathrm{TeV}$. The various contributions to the numerator and the resulting values of $A_{C}\left(y_{c}=1\right)$ at $7 \mathrm{TeV}$ center-ofmass energy are given in Table 4. The size of the $O\left(\alpha \alpha_{s}^{2}\right)$ mixed QCD-QED corrections to $q \bar{q}$ initiated contributions relative to those of $O\left(\alpha_{s}^{3}\right)$ QCD is now $\sim 13 \%$, which, as already mentioned in Sect. 2.1, is due to the fact that the ratio of $u \bar{u}$ versus $d \bar{d}$ annihilation is 2:1 at the LHC as compared to $4: 1$ for $p \bar{p}$ collisions. The size of the $O\left(\alpha_{s}^{3}\right)$ QCD contributions from $q g$ fusion amount to about $5 \%\left(M_{c}=2 m_{t}\right)$ of the $q \bar{q}$ contributions. At $\sqrt{s}=14 \mathrm{TeV}$ and $M_{c}=1 \mathrm{TeV}$, they rise to $\sim 17 \%$. Here, and also for all other LHC asymmetries discussed below, we take into account also the mixed QCD-QED corrections of $O\left(\alpha \alpha_{s}^{2}\right)$ to $q g \rightarrow t \bar{t} q$ which are of the same order of magnitude as the mixed QCD-weak corrections of $O\left(\alpha \alpha_{s}^{2}\right)$, as shown in Table 4. The size of these corrections can be easily understood. By diagram inspection at the level of initial partons one obtains that the ratio $f_{q}=O\left(\alpha \alpha_{s}^{2}\right)_{Q E D} / O\left(\alpha_{s}^{3}\right)$ for $q g \rightarrow t \bar{t} q$ is given by

$$
f_{q}=\frac{4 \alpha Q_{q} Q_{t}}{\alpha_{s} d_{a b c}^{2} / 4}=\frac{24 \alpha Q_{q} Q_{t}}{5 \alpha_{s}},
$$

where $d_{a b c}^{2}=40 / 3$. For $p p$ collisions at the LHC one gets therefore the ratio

$$
f^{Q E D}=\frac{4 f_{u}+2 f_{d}}{6}=\frac{16 \alpha}{15 \alpha_{s}} .
$$


Using $\alpha_{s} \simeq 0.11$ and $\alpha \simeq 0.008$, one gets $f^{Q E D} \simeq 0.078$. This estimate explains the respective results of Table 4 which were obtained by integrating the respective matrix elements and PDF.

In Table 5 the values of $A_{C}\left(y_{c}=1\right)$ are given for $\sqrt{s}=7,8$, and $14 \mathrm{TeV}$, both for QCD and for QCD plus electroweak contributions. The given uncertainties are due to scale variations. As above we choose $\mu=m_{t} / 2, m_{t}$, and $2 m_{t}$. The asymmetry $A_{C}\left(y_{c}=1\right)$ increases with increasing lower bound $M_{c}$ on $M_{t \bar{t}}$. But, as the numbers for the denominator $D_{C}$ in Table 4 show, the event numbers decrease rapidly with increasing $M_{c}$. The ratio of the electroweak and QCD contributions to $A_{C}\left(y_{c}=1\right.$ ) is $13 \%$ for $\sqrt{s}=7 \mathrm{TeV}$ (no cut on $M_{t \bar{t}}$ ) and increases slightly to $16 \%$ for $\sqrt{s}=14 \mathrm{TeV}$ and $M_{t \bar{t}}>1 \mathrm{TeV}$.

The various contributions to the numerator of the edge asymmetry are collected in Table 6 for $\sqrt{s}=7 \mathrm{TeV}$, and $A_{E}\left(y_{c}\right)$ is given as a function of $y_{c}$ for $\sqrt{s}=7,8$ and $14 \mathrm{TeV}$ in Table 7 . The statistical significances of $A_{E}$ and $A_{C}$ are of comparable size. For instance, $A_{E}\left(y_{c}=1\right)=1 \%$ while $A_{C}\left(y_{c}=1\right)=-0.6 \%$ at $7 \mathrm{TeV}$. The smaller value of $A_{C}$ is compensated by the larger number of events with $y_{c} \leq 1$.

\begin{tabular}{|c|c|c|c|c|c|}
\hline \multicolumn{2}{|c|}{$M_{c}$} & $2 m_{t}$ & $0.5 \mathrm{TeV}$ & $0.7 \mathrm{TeV}$ & $1 \mathrm{TeV}$ \\
\cline { 1 - 5 }$N_{C}(\mathrm{pb})$ & & & & \\
\hline \multirow{2}{*}{$O\left(\alpha_{s}^{3}\right)$} & $q \bar{q}$ & -0.6270 & -0.3718 & -0.1202 & $-2.274 \times 10^{-2}$ \\
\cline { 2 - 6 } & $q g$ & -0.0379 & -0.0227 & -0.0100 & -0.0020 \\
\hline$O\left(\alpha^{2}\right)_{\text {weak }}$ & $q \bar{q}$ & -0.0234 & -0.0134 & -0.0040 & $-6 \times 10^{-4}$ \\
\hline \multirow{2}{*}{$O\left(\alpha \alpha_{s}^{2}\right)_{\text {weak }}$} & $q \bar{q}$ & $-2.5 \times 10^{-3}$ & $-1.3 \times 10^{-3}$ & $-4.5 \times 10^{-4}$ & $-9 \times 10^{-5}$ \\
\cline { 2 - 6 } & $q g$ & $7.1 \times 10^{-3}$ & $1.7 \times 10^{-3}$ & $-4.4 \times 10^{-4}$ & $-2.1 \times 10^{-4}$ \\
\hline \multirow{2}{*}{$O\left(\alpha \alpha_{s}^{2}\right)_{Q E D}$} & $q \bar{q}$ & $-7.85 \times 10^{-2}$ & $-4.81 \times 10^{-2}$ & $-1.53 \times 10^{-2}$ & $-2.7 \times 10^{-3}$ \\
\cline { 2 - 6 } & $q g$ & $-2.7 \times 10^{-3}$ & $-2.0 \times 10^{-3}$ & $-7 \times 10^{-4}$ & $-1 \times 10^{-4}$ \\
\hline \multicolumn{2}{c|}{ Total } & -0.7648 & -0.4576 & -0.1512 & -0.0286 \\
\hline \hline$D_{Q C D}^{L O}(\mathrm{pb})$ & 126.76 & 45.76 & 9.89 & 1.35 \\
\hline \hline$A_{C}(\%)$ & -0.60 & -1.00 & -1.53 & -2.13 \\
\hline
\end{tabular}

Table 4: The contributions to the numerator and denominator of $A_{C}\left(y_{c}=1\right)$, defined in (4), for $\mu=m_{t}$ at the $\mathrm{LHC}(7 \mathrm{TeV})$.

The center and edge asymmetries were computed before in [16] at NLO QCD including electroweak corrections, as functions of $y_{c}$ for 7 and $14 \mathrm{TeV}$. Ref. [16] evaluated the numerators and denominators of the asymmetries with the PDF set [48] and took the purely weak corrections only approximately into account. Our results above agre 5 , within the given uncertainties, with [16].

\footnotetext{
${ }^{5}$ The definition of the central asymmetry $A_{C}$ in (4) differs by a sign from that of [16].
} 


\begin{tabular}{|c|cc|c|c|c|c|}
\hline$\sqrt{s}$ & & & $M_{c}=2 m_{t}$ & $0.5 \mathrm{TeV}$ & $0.7 \mathrm{TeV}$ & $1 \mathrm{TeV}$ \\
\hline $7 \mathrm{TeV}$ & QCD: & $A_{C}(\%)$ & $-0.53(3)$ & $-0.86(3)$ & $-1.32(5)$ & $-1.77(7)$ \\
& QCD + EW: & $A_{C}(\%)$ & $-0.60(3)$ & $-1.00(4)$ & $-1.53(5)$ & $-2.07(7)$ \\
\hline $8 \mathrm{TeV}$ & QCD: & $A_{C}(\%)$ & $-0.47(2)$ & $-0.76(2)$ & $-1.18(4)$ & $-1.66(5)$ \\
& QCD + EW: & $A_{C}(\%)$ & $-0.54(3)$ & $-0.88(4)$ & $-1.37(4)$ & $-1.94(5)$ \\
\hline \hline & & & $M_{c}=2 m_{t}$ & $0.5 \mathrm{TeV}$ & $1 \mathrm{TeV}$ & $2 \mathrm{TeV}$ \\
\hline $14 \mathrm{TeV}$ & QCD: & $A_{C}(\%)$ & $-0.26(2)$ & $-0.45(2)$ & $-1.09(4)$ & $-1.90(6)$ \\
& QCD + EW: & $A_{C}(\%)$ & $-0.30(3)$ & $-0.52(4)$ & $-1.29(5)$ & $-2.21(5)$ \\
\hline
\end{tabular}

Table 5: The central charge asymmetry $A_{C}\left(y_{c}=1\right)$ for the LHC at 7,8, and $14 \mathrm{TeV}$, for events with $M_{t \bar{t}} \geq M_{c}$. The uncertainties are due to scale variations.

\begin{tabular}{|c|c|c|c|c|}
\hline \multicolumn{2}{|c|}{$N_{E}(\mathrm{pb})$} & $Y_{C}=0.5$ & $Y_{C}=1$ & $Y_{C}=2$ \\
\hline \multirow{2}{*}{$O\left(\alpha_{s}^{3}\right)$} & $q \bar{q}$ & 0.4325 & 0.6270 & 0.3117 \\
\cline { 2 - 5 } & $q g$ & 0.0238 & 0.0379 & 0.0125 \\
\hline$O\left(\alpha^{2}\right)_{\text {weak }}$ & $q \bar{q}$ & 0.0154 & 0.0234 & 0.0103 \\
\hline \multirow{2}{*}{$O\left(\alpha \alpha_{s}^{2}\right)_{\text {weak }}$} & $q \bar{q}$ & $1.2 \times 10^{-3}$ & $2.6 \times 10^{-3}$ & $2.1 \times 10^{-3}$ \\
\cline { 2 - 5 } & $q g$ & $-4.6 \times 10^{-3}$ & $-7.1 \times 10^{-3}$ & $-4.1 \times 10^{-3}$ \\
\hline \multirow{2}{*}{$O\left(\alpha \alpha_{s}^{2}\right)_{Q E D}$} & $q \bar{q}$ & 0.0488 & 0.0785 & 0.0450 \\
\cline { 2 - 5 } & $q g$ & $1.8 \times 10^{-3}$ & $2.7 \times 10^{-3}$ & $1.1 \times 10^{-3}$ \\
\hline Total & 0.5189 & 0.7648 & 0.3776 \\
\hline$D_{Q C D}^{L O}(\mathrm{pb})$ & 131.86 & 73.77 & 10.27 \\
\hline$A_{E}(\%)$ & 0.39 & 1.04 & 3.69 \\
\hline
\end{tabular}

Table 6: The contributions to the numerator and denominator of $A_{E}$, defined in (5), for $\mu=m_{t}$ at the $\mathrm{LHC}(7 \mathrm{TeV})$.

\section{Cut-independent charge asymmetries}

The CMS [13,14] and ATLAS [15] experiments measured the following rapidity-cut independent charge asymmetries:

$$
\begin{aligned}
& A_{C}^{\Delta|y|}=\frac{N(\Delta|y|>0)-N(\Delta|y|<0)}{N(\Delta|y|>0)+N(\Delta|y|<0)}, \\
& A_{C}^{\Delta|\eta|}=\frac{N(\Delta|\eta|>0)-N(\Delta|\eta|<0)}{N(\Delta|\eta|>0)+N(\Delta|\eta|<0)},
\end{aligned}
$$

where $\Delta|y|=\left|y_{t}\right|-\left|y_{\bar{t}}\right|$ and likewise for the pseudorapidities, $\Delta|\eta|=\left|\eta_{t}\right|-\left|\eta_{\bar{t}}\right|$, in the laboratory frame.

We compute these asymmetries for $t \bar{t}$ events with $M_{t t} \geq M_{c}$. As above, we choose $M_{c}=2 m_{t}$ (i.e., all events), $0.5 \mathrm{TeV}, 0.7 \mathrm{TeV}$ and $1 \mathrm{TeV}$. Our NLO QCD predictions and those including the electroweak corrections are given in Tables 8 and 9 for $\sqrt{s}=7,8$, and $14 \mathrm{TeV}$. With a cut $M_{t t} \geq 1 \mathrm{TeV}$, the asymmetries $A_{C}^{\Delta|y|}, A_{C}^{\Delta|\eta|}$ increase by a factor of about two. The ratio of electroweak and QCD contributions to the asymmetries is $15 \%$ for $\sqrt{s}=7 \mathrm{TeV}$ and no cut on $M_{t \bar{t}}$, and it increases to $\gtrsim 20 \%$ at $\sqrt{s}=14 \mathrm{TeV}$ and large $M_{t \bar{t}}$.

The asymmetries $A_{C}^{\Delta|y|}$ and $A_{C}^{\Delta|\eta|}$ were computed also in [16] in the SM without a cut on $M_{t \bar{t}}$. The respective numbers in Tables 8 and 9 agree with these results. 


\begin{tabular}{|c|cc|c|c|c|}
\hline$\sqrt{s}$ & & & $y_{c}=0.5$ & $y_{c}=1$ & $y_{c}=2$ \\
\hline $7 \mathrm{TeV}$ & QCD: & $A_{E}(\%)$ & $0.35(1)$ & $0.90(3)$ & $3.16(6)$ \\
& QCD + EW: & $A_{E}(\%)$ & $0.39(2)$ & $1.04(4)$ & $3.69(7)$ \\
\hline $8 \mathrm{TeV}$ & QCD: & $A_{E}(\%)$ & $0.29(1)$ & $0.74(3)$ & $2.69(6)$ \\
& QCD + EW: & $A_{E}(\%)$ & $0.31(2)$ & $0.86(3)$ & $3.24(6)$ \\
\hline $14 \mathrm{TeV}$ & QCD: & $A_{E}(\%)$ & $0.12(1)$ & $0.32(1)$ & $1.28(5)$ \\
& QCD + EW: & $A_{E}(\%)$ & $0.14(1)$ & $0.37(3)$ & $1.49(9)$ \\
\hline
\end{tabular}

Table 7: The edge asymmetry $A_{E}$ as a function of $y_{c}$ for the LHC at 7,8, and $14 \mathrm{TeV}$. The uncertainties are due to scale variations.

\begin{tabular}{|c|cc|c|c|c|c|}
\hline$\sqrt{s}$ & & & $M_{c}=2 m_{t}$ & $0.5 \mathrm{TeV}$ & $0.7 \mathrm{TeV}$ & $1 \mathrm{TeV}$ \\
\hline $7 \mathrm{TeV}$ & QCD: & $A_{C}^{\Delta|y|}(\%)$ & $1.07(4)$ & $1.27(4)$ & $1.68(4)$ & $2.06(5)$ \\
& QCD + EW: & $A_{C}^{\Delta|y|}(\%)$ & $1.23(5)$ & $1.48(4)$ & $1.95(4)$ & $2.40(6)$ \\
\hline $8 \mathrm{TeV}$ & QCD: & $A_{C}^{\Delta|y|}(\%)$ & $0.96(4)$ & $1.14(4)$ & $1.48(4)$ & $1.85(4)$ \\
& QCD + EW: & $A_{C}^{\Delta|y|}(\%)$ & $1.11(4)$ & $1.33(5)$ & $1.73(5)$ & $2.20(5)$ \\
\hline \hline & & & $M_{c}=2 m_{t}$ & $0.5 \mathrm{TeV}$ & $1 \mathrm{TeV}$ & $2 \mathrm{TeV}$ \\
\hline $14 \mathrm{TeV}$ & QCD: & $A_{C}^{\Delta|y|}(\%)$ & $0.58(3)$ & $0.74(3)$ & $1.11(5)$ & $1.72(10)$ \\
& QCD + EW: & $A_{C}^{\Delta|y|}(\%)$ & $0.67(4)$ & $0.86(5)$ & $1.32(8)$ & $2.12(10)$ \\
\hline
\end{tabular}

Table 8: The charge asymmetry $A_{C}^{\Delta|y|}$ defined in (8) at the LHC, for $M_{t \bar{t}} \geq M_{c}$.

The experimental results of the CMS and ATLAS collaborations are given in Table 10. The results agree, within the present uncertainties, with the SM predictions given above6. The recent CMS analysis [14, based on a data sample of $L_{\text {int }}=4.7 \mathrm{fb}^{-1}$, measured the charge asymmetry $A_{C}^{\Delta|y|}$ also differentially; in particular as a function of $M_{t \bar{t}}$. The respective data given in [14] agree, within the still large experimental errors, with our SM prediction of the $M_{t \bar{t}}$ dependence of $A_{C}^{\Delta|y|}$ given in Table 8 ,

\section{Boosted charge asymmetry}

Another way to enhance the $t \bar{t}$ charge asymmetries at the LHC is to select $t \bar{t}$ events whose center-of-mass frame has a considerable Lorentz boost with respect to the beam axis. The velocity of the $t \bar{t}$ system along the beam axis is given by

$$
\beta=\frac{\left|p_{t}^{z}+p_{\bar{t}}^{z}\right|}{E_{t}+E_{\bar{t}}}
$$

\footnotetext{
${ }^{6}$ In view of the positive charge asymmetry measured at the Tevatron one expects the LHC asymmetry $A_{C}$ to be positive, too, within the SM. However, there are examples of new physics models which yield a negative LHC asymmetry; see, e.g., [54].
} 


\begin{tabular}{|c|c|c|c|c|c|c|}
\hline$\sqrt{s}$ & & & $M_{c}=2 m_{t}$ & $0.5 \mathrm{TeV}$ & $0.7 \mathrm{TeV}$ & $1 \mathrm{TeV}$ \\
\hline \multirow[t]{2}{*}{$7 \mathrm{TeV}$} & QCD: & $A_{C}^{\Delta|\eta|}(\%)$ & $1.36(6)$ & $1.39(5)$ & $1.76(5)$ & $2.15(5)$ \\
\hline & $\mathrm{CD}+\mathrm{E}$ & $A_{C}^{\Delta|\eta|}(\%)$ & $1.56(7)$ & $1.64(6)$ & $2.06(5)$ & $2.52(5)$ \\
\hline \multirow[t]{3}{*}{$8 \mathrm{TeV}$} & QCD: & $A_{C}^{\Delta|\eta|}(\%)$ & 1. & $1.25(5)$ & $1.56(4)$ & $1.93(4)$ \\
\hline & $\mathrm{CD}+\mathrm{E}$ & $A_{C}^{\Delta|\eta|}(\%)$ & 1.4 & 1.47 & $1.84(5)$ & $2.30(5)$ \\
\hline & & & $\bar{c}_{M_{c}}=2 m_{t}$ & $0.5 \mathrm{TeV}$ & 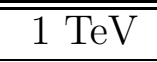 & $2 \mathrm{TTeV}$ \\
\hline \multirow[t]{2}{*}{$14 \mathrm{TeV}$} & & & & 0.8 & $1.16(5)$ & $1.82(7)$ \\
\hline & $\mathrm{QCD}+\mathrm{EW}:$ & $A_{C}^{\Delta|\eta|}(\%)$ & $0.96(6)$ & $1.00(6)$ & $1.44(6)$ & $2.38(8)$ \\
\hline
\end{tabular}

Table 9: The charge asymmetry $A_{C}^{\Delta|\eta|}$ defined in (9) at the LHC, for $M_{t \bar{t}} \geq M_{c}$.

\begin{tabular}{|c|c|c|}
\hline & $A_{C}^{\Delta|y|}(\%)$ & $A_{C}^{\Delta|\eta|}(\%)$ \\
\hline CMS & $0.4 \pm 1.0 \pm 1.2[14]$ & $-1.7 \pm 3.2_{-3.6}^{+2.5}[13]$ \\
\hline ATLAS & $-1.8 \pm 2.8 \pm 2.3[15]$ & \\
\hline
\end{tabular}

Table 10: CMS [13,14] and ATLAS [15] results at the LHC (7 TeV).

where $p^{z}$ and $E$ is the corresponding longitudinal momentum component and energy in the laboratory frame, respectively. Ref. [21] proposed to evaluate the asymmetries (8), (9) for $t \bar{t}$ events with $\beta$ larger than a certain minimal value $\beta_{\min }$. By increasing $\beta_{\min }$ the $q \bar{q}$ initiated $t \bar{t}$ sample and therefore the $t \bar{t}$ charge asymmetry grows.

This 'boosted charge asymmetry' is similar to the one-sided charge asymmetry [17] that will be discussed below. Using the variable $\beta$ rather than the longitudinal momentum $\left|p_{t}^{z}+p_{t}^{z}\right|$ has obvious experimental advantages: the ratio $\beta$ is less affected by uncertainties due to jet energy scale and resolution.

Table 11 contains our results for the charge asymmetry (9) as a function of $\beta_{\text {min }}$, i.e. $A_{C}^{\Delta|\eta|}\left(\beta_{\text {min }}\right)$, for the LHC at 7,8 , and $14 \mathrm{TeV}$. As expected the SM-induced asymmetry increases with increasing $\beta_{\min }$ - of course, again at the expense of decreasing $t \bar{t}$ samples. The contribution of the electroweak interactions is about $15 \%$ compared to the pure QCD asymmetry.

\section{One-sided charge asymmetry}

Finally, we consider an asymmetry introduced and computed within QCD in [17]. Let $P_{t \bar{t}}^{z}$ be the component along the beam of the sum of the $t$ and $\bar{t}$ momenta, $\mathbf{P}_{t \bar{t}}=\mathbf{p}_{t}+\mathbf{p}_{\bar{t}}$, in the laboratory frame. For $p p$ collisions, a non-zero charge asymmetry may be obtained by selecting $t \bar{t}$ events with $P_{t \bar{t}}^{z}>0$, or events with $P_{t \bar{t}}^{z}<0$. One may define a one-sided charge asymmetry by

$$
A_{O}=\left.\frac{N(\Delta y>0)-N(\Delta y<0)}{N(\Delta y>0)+N(\Delta y<0)}\right|_{P_{t t}^{z}>P_{c}^{z}}=\left.\frac{N(\Delta y<0)-N(\Delta y>0)}{N(\Delta y>0)+N(\Delta y<0)}\right|_{P_{t t}^{z}<-P_{c}^{z}},
$$

where $\Delta y=y_{t}-y_{\bar{t}}$ in the laboratory frame.

Similar to the boosted asymmetry discussed above, the fraction of the $q \bar{q}$ initiated $t \bar{t}$ sample and hence $A_{O}$ is increased by increasing the lower cut $P_{c}^{z}$ on $P_{t \bar{t}}^{z}$. A further, more moderate enhancement can be achieved by applying the additional cut $M_{t \bar{t}} \geq M_{c}$. 


\begin{tabular}{|c|c|c|c|c|}
\hline \multicolumn{2}{|c|}{$A_{C}^{\Delta|\eta|}(\%)$} & $7 \mathrm{TeV}$ & $8 \mathrm{TeV}$ & $14 \mathrm{TeV}$ \\
\hline \hline$\beta_{\text {min }}=0.1$ & QCD & $1.41(7)$ & $1.28(5)$ & $0.86(5)$ \\
& QCD+EW & $1.62(7)$ & $1.48(7)$ & $1.00(7)$ \\
\hline$\beta_{\text {min }}=0.2$ & QCD & $1.50(7)$ & $1.37(6)$ & $0.90(5)$ \\
& QCD+EW & $1.72(8)$ & $1.57(7)$ & $1.04(7)$ \\
\hline$\beta_{\text {min }}=0.3$ & QCD & $1.63(7)$ & $1.47(6)$ & $0.95(5)$ \\
& QCD+EW & $1.87(8)$ & $1.69(8)$ & $1.10(7)$ \\
\hline$\beta_{\text {min }}=0.4$ & QCD & $1.77(8)$ & $1.56(7)$ & $1.01(6)$ \\
& QCD+EW & $2.02(9)$ & $1.79(8)$ & $1.17(8)$ \\
\hline$\beta_{\text {min }}=0.5$ & QCD & $1.87(10)$ & $1.69(7)$ & $1.10(6)$ \\
& QCD+EW & $2.16(10)$ & $1.95(9)$ & $1.27(8)$ \\
\hline$\beta_{\text {min }}=0.6$ & QCD & $2.07(10)$ & $1.86(8)$ & $1.21(6)$ \\
& QCD+EW & $2.38(10)$ & $2.14(10)$ & $1.39(9)$ \\
\hline$\beta_{\text {min }}=0.7$ & QCD & $2.30(10)$ & $2.08(8)$ & $1.33(7)$ \\
& QCD+EW & $2.65(11)$ & $2.40(11)$ & $1.53(10)$ \\
\hline$\beta_{\text {min }}=0.8$ & QCD: & $2.67(12)$ & $2.39(10)$ & $1.54(9)$ \\
& QCD+EW & $3.08(12)$ & $2.76(12)$ & $1.78(11)$ \\
\hline$\beta_{\text {min }}=0.9$ & QCD & $3.22(13)$ & $2.95(12)$ & $1.90(10)$ \\
& QCD+EW & $3.74(12)$ & $3.42(13)$ & $2.20(12)$ \\
\hline
\end{tabular}

Table 11: The charge asymmetry $A_{C}^{\Delta|\eta|}$ defined in (9) for $t \bar{t}$ events with $\beta=$ $\left|p_{t}^{z}+p_{\bar{t}}^{z}\right| /\left(E_{t}+E_{\bar{t}}\right)>\beta_{\min }$ at the LHC.

In Table 12 we collect our SM results for $A_{O}$ as a function of $P_{c}^{z}$, for $M_{c}=2 m_{t}$ and 0.5 $\mathrm{TeV}$, for the LHC at 7,8, and $14 \mathrm{TeV}$. In the case of $A_{O}$ the ratio of electroweak and QCD contributions remains essentially constant if $P_{c}^{z}$ and/or $M_{c}$ is increased: for the results given in Table 12 this ratio is between 15 and $17 \%$.

The results for the one-sided asymmetry at NLO QCD given in [17] are systematically larger than the corresponding QCD results given in Table 12. This is due to the fact that in [17] the denominator of $A_{O}$ was evaluated with LO matrix elements and LO PDF which leads to a smaller denominator than in our case.

Finally we emphasize that the magnitudes of all LHC charge asymmetries discussed in this section, especially for large $M_{t \bar{t}}, \beta_{\min }$, or $P_{c}^{z}$ cuts depend sensitively on how the denominators of the asymmetries are evaluated. If one computes these denominators in 'Monte Carlo fashion' with NLO matrix elements, the magnitudes of the asymmetries decrease significantly. Thus the scale uncertainties given in the above tables underestimate the true theory uncertainties, which are rather of the order of $\sim 30 \%$.

\section{Leptonic forward-backward and charge asymmetries}

The asymmetries at the level of the intermediate $t \bar{t}$ states considered in the previous section cannot be measured directly, but are extracted from the data on dileptonic and lepton plus jets final states by an unfolding procedure. On the other hand, the top-quark forwardbackward and charge asymmetries lead also to asymmetries for the daughter leptons from 


\begin{tabular}{|c|c|c|c|c|c|c|c|}
\hline$\sqrt{s}$ & $M_{C}$ & $P_{c}^{z}(\mathrm{GeV})$ & 0 & 250 & 500 & 750 & 1000 \\
\hline \multirow[t]{4}{*}{$7 \mathrm{TeV}$} & \multirow[t]{2}{*}{$2 m_{t}$} & $\overline{\mathrm{QCD}} A_{O}(\%)$ & $1.13(4)$ & $1.50(5)$ & $2.10(6)$ & $2.62(8)$ & $3.02(8)$ \\
\hline & & $\mathrm{QCD}+\mathrm{EW} A_{O}(\%)$ & $1.30(5)$ & $1.74(7)$ & $2.43(7)$ & $3.06(9)$ & $3.55(9)$ \\
\hline & \multirow[t]{2}{*}{$500 \mathrm{GeV}$} & $\mathrm{QCD} A_{O}(\%)$ & $1.31(4)$ & $1.75(5)$ & $2.38(5)$ & $2.95(5)$ & $3.47(4)$ \\
\hline & & $\mathrm{QCD}+\mathrm{EW} A_{O}(\%)$ & $1.53(5)$ & $2.04(6)$ & $2.77(6)$ & $3.45(5)$ & $4.09(3)$ \\
\hline \multirow[t]{4}{*}{$8 \mathrm{TeV}$} & \multirow{2}{*}{$2 m_{t}$} & $\mathrm{QCD} A_{O}(\%)$ & $1.02(4)$ & $1.35(4)$ & $1.84(6)$ & $2.39(8)$ & $2.75(9)$ \\
\hline & & $\mathrm{QCD}+\mathrm{EW} A_{O}(\%)$ & $1.17(5)$ & $1.55(6)$ & $2.13(7)$ & $2.78(8)$ & $3.23(9)$ \\
\hline & \multirow[t]{2}{*}{$500 \mathrm{GeV}$} & $\mathrm{QCD} A_{O}(\%)$ & $1.15(3)$ & $1.53(4)$ & $2.07(5)$ & $2.61(5)$ & $3.08(6)$ \\
\hline & & $\mathrm{QCD}+\mathrm{EW} A_{O}(\%)$ & $1.34(4)$ & $1.77(5)$ & $2.40(5)$ & $3.04(6)$ & $3.61(5)$ \\
\hline \multirow[t]{4}{*}{$14 \mathrm{TeV}$} & \multirow[t]{2}{*}{$2 m_{t}$} & $\mathrm{QCD} A_{O}(\%)$ & $0.61(2)$ & $0.79(3)$ & $1.07(4)$ & $1.27(6)$ & $1.57(5)$ \\
\hline & & $\mathrm{QCD}+\mathrm{EW} A_{O}(\%)$ & $0.70(5)$ & $0.91(7)$ & $1.23(8)$ & $1.48(8)$ & $1.83(9)$ \\
\hline & \multirow[t]{2}{*}{$500 \mathrm{GeV}$} & $\mathrm{QCD} A_{O}(\%)$ & $0.74(3)$ & $0.92(3)$ & $1.18(4)$ & $1.44(6)$ & $1.74(6)$ \\
\hline & & $\mathrm{QCD}+\mathrm{EW} A_{O}(\%)$ & $0.86(6)$ & $1.07(7)$ & $1.38(9)$ & $1.68(9)$ & $2.04(8)$ \\
\hline
\end{tabular}

Table 12: The one-sided asymmetry $A_{O}$ defined in (11) as a function of $P_{c}^{z}$ without and with an additional cut on $M_{t \bar{t}}$ for the $\mathrm{LHC}$ at 7,8 , and $14 \mathrm{TeV}$.

semileptonic top-quark decay. Although these asymmetries are expected to be smaller than the corresponding ones for top quarks, because the lepton does not strictly follow the direction of its quark parent, the leptonic asymmetries should be measurable more precisely and should allow for a more direct comparison between theory and experiment.

We consider here, for the Tevatron and for the LHC, dileptonic final states resulting from an intermediate $t \bar{t}$ state:

$$
p \bar{p}, p p \rightarrow t \bar{t}+X \rightarrow \ell^{+} \ell^{\prime-} j_{b} j_{\bar{b}}+X,
$$

where $\ell=e, \mu$ and $j_{b}$ denotes a $b$ jet.

We compute the leptonic asymmetries defined below without and with acceptance cuts. For the dileptonic final states we use the following cuts $\left(\ell=e, \mu, E_{T}^{\text {miss }}\right.$ denotes the missing transverse energy, and $\eta$ is the pseudorapidity):

Tevatron: $\quad p_{T}^{\ell} \geq 20 \mathrm{GeV}, \quad\left|\eta_{\ell}\right| \leq 2.0, \quad p_{T}^{j} \geq 20 \mathrm{GeV}, \quad\left|\eta_{j}\right| \leq 2.0, \quad E_{T}^{\text {miss }} \geq 25 \mathrm{GeV}$, (13)

$$
\text { LHC : } \quad p_{T}^{\ell} \geq 20 \mathrm{GeV}, \quad\left|\eta_{\ell}\right| \leq 2.5, \quad p_{T}^{j} \geq 25 \mathrm{GeV}, \quad\left|\eta_{j}\right| \leq 2.4, \quad E_{T}^{\text {miss }} \geq 60 \mathrm{GeV} .
$$

The index $j$ refers to a $b, \bar{b}$, a light (anti)quark, or a gluon jet, and we specify in the following how we apply these cuts.

Our results for the Tevatron asymmetry $A^{\ell}$ without cuts given below apply also to the lepton plus jets events at the Tevatron.

$$
p \bar{p} \rightarrow t \bar{t}+X \rightarrow \ell^{+} j_{b} j_{\bar{b}} j_{1} j_{2}+X, \quad \ell^{-} j_{\bar{b}} j_{b} j_{1} j_{2}+X,
$$

where $j_{1,2}$ denote non- $b$ jets.

\section{Tevatron}

For the Tevatron one can define for both types of final states (12), (15) a leptonic charge asymmetry. Let $N_{\ell^{ \pm}}\left(\eta_{\ell^{ \pm}}\right)$be the number of $t \bar{t}$ events that contain a positively/negatively 
charged lepton $\ell^{ \pm}$with pseudorapidity $\eta_{\ell^{ \pm}}$in the laboratory frame. One may consider the leptonic charge asymmetry

$$
A^{\ell}=\frac{N_{\ell^{+}}\left(\eta_{\ell^{+}}>0\right)-N_{\ell^{-}}\left(\eta_{\ell^{-}}>0\right)}{N_{\ell^{+}}\left(\eta_{\ell^{+}}>0\right)+N_{\ell^{-}}\left(\eta_{\ell^{+}}>0\right)} .
$$

If CP invariance holds, then $N_{\ell^{+}}\left(\eta_{\ell^{+}}\right)=N_{\ell^{-}}\left(-\eta_{\ell^{-}}\right)$and $A^{\ell}$ is equal to the leptonic forwardbackward asymmetry, $A^{\ell}=A_{F B}^{\ell^{+}}=-A_{F B}^{\ell^{-}}$, where

$$
A_{F B}^{\ell^{ \pm}}=\frac{N_{\ell^{ \pm}}\left(\eta_{\ell^{ \pm}}>0\right)-N_{\ell^{ \pm}}\left(\eta_{\ell^{ \pm}}<0\right)}{N_{\ell^{ \pm}}\left(\eta_{\ell^{ \pm}}>0\right)+N_{\ell^{ \pm}}\left(\eta_{\ell^{ \pm}}<0\right)} .
$$

In analogy to the $t \bar{t}$ pair asymmetry $A^{t \bar{t}}$ one may consider, for dileptonic final states, the leptonic pair asymmetry

$$
A^{\ell \ell}=\frac{N_{\ell \ell}\left(\Delta \eta_{\ell}>0\right)-N_{\ell \ell}\left(\Delta \eta_{\ell}<0\right)}{N_{\ell \ell}\left(\Delta \eta_{\ell}>0\right)+N_{\ell \ell}\left(\Delta \eta_{\ell}<0\right)},
$$

where $\Delta \eta_{\ell}=\eta_{\ell^{+}}-\eta_{\ell^{-}}$. In analogy to $A^{t \bar{t}}$ versus $A_{F B}^{t}$, the pair asymmetry $A^{\ell \ell}$ is, for kinematical reasons, larger than $A^{\ell}$.

We compute the asymmetries (17) and (18) at NLO QCD with respect to $t \bar{t}$ production and $t$ and $\bar{t}$ decay, including the electroweak corrections to $t \bar{t}$ production as described in Sect. 2.1 and 2.2 . The $t \bar{t}$ spin correlations are taken into account. The radiative corrections were implemented into our computer code as described in [12]. In computing the ratios (16) - (18) we use the same procedure as in Sect. 2.1 and 2.2, namely, we use in the denominator LO matrix elements and the NLO PDF set CTEQ6.6M both in the numerator and denominator. We calculated the above Tevatron observables inclusively as follows. For the dileptonic events, which at NLO in the gauge couplings contain at most 3 partons in the final state, we require that at least 2 partons satisfy the above dileptonic cuts. We checked that the results do not change when using instead the $k_{\perp}$ jet algorithm [49]. This is to say we checked an inclusive calculation against $\ell^{+} \nu_{\ell} \ell^{-} \bar{\nu}_{\ell} j_{b} j_{\bar{b}}$ (LO) and $\ell^{+} \nu_{\ell} \ell^{-} \bar{\nu}_{\ell} j_{b} j_{\bar{b}} j$ (NLO), where $j$ denotes a gluon or light quark jet.

Our results for $A^{\ell}=A_{F B}^{\ell^{+}}$and $A^{\ell \ell}$ are collected in Table 13. As expected, in the SM 7 the leptonic charge asymmetry $A^{\ell}$ has the same sign as the top-quark charge asymmetry given in Sect. 2.1, but is smaller in magnitude, while $A^{\ell \ell}$ is larger than $A^{\ell}$ but smaller than $A^{t \bar{t}}$. As is the case for the $t \bar{t}$ charge asymmetry, selecting events with large rapidity difference $\left|\Delta y_{\ell}\right|$ or large $M_{t \bar{t}}$ increases $A_{\ell}$ and $A^{\ell \ell}$ significantly.

The results in the last column of Table 13 were obtained without applying selection cuts. These numbers can be directly compared to experimental results that are corrected for detector effects, background contributions, and acceptances. As one can see from Table 13, removing the cuts has only a minor effect on the asymmetries.

Our results for $A^{\ell}$ without cuts in the last column of Table 13 apply also to the lepton + jets final states (15) at the Tevatron.

\footnotetext{
${ }^{7}$ The asymmetries $A^{\ell}$ and $A^{\ell \ell}$, when measured close to the $t \bar{t}$ production threshold, may contain information independent from the inclusive lepton asymmetries [51.
} 


\begin{tabular}{|c|c|c|c|}
\hline & & with cuts & without cuts \\
\hline$A^{\ell}(\%)$ & QCD: & $3.0(3)$ & $3.1(3)$ \\
& QCD + EW: & $3.6(2)$ & $3.8(3)$ \\
\hline$A^{\ell}(\%)$ & QCD: & $5.2(5)$ & $5.8(5)$ \\
$\left(M_{t \bar{t}} \geq 450 \mathrm{GeV}\right)$ & QCD + EW: & $6.4(5)$ & $7.0(5)$ \\
\hline$A^{\ell}(\%)$ & QCD: & $1.6(1)$ & $1.5(1)$ \\
$\left(M_{t \bar{t}}<450 \mathrm{GeV}\right)$ & QCD + EW: & $1.9(1)$ & $1.8(1)$ \\
\hline$A^{\ell \ell}(\%)$ & QCD: & $4.0(4)$ & $4.0(4)$ \\
& QCD + EW: & $4.8(4)$ & $4.8(4)$ \\
\hline$A^{\ell \ell}(\%)$ & QCD: & $7.0(6)$ & $6.3(6)$ \\
$\left(\left|\Delta y_{\ell}\right| \geq 1\right)$ & QCD + EW: & $8.5(6)$ & $7.5(6)$ \\
\hline$A^{\ell \ell}(\%)$ & QCD: & $1.9(2)$ & $1.6(1)$ \\
$\left(\left|\Delta y_{\ell}\right|<1\right)$ & QCD + EW: & $2.3(2)$ & $1.9(2)$ \\
\hline$A^{\ell \ell}(\%)$ & QCD: & $6.7(5)$ & $7.1(6)$ \\
$\left(M_{t \bar{t}} \geq 450 \mathrm{GeV}\right)$ & QCD + EW: & $8.2(5)$ & $8.7(6)$ \\
\hline$A^{\ell \ell}(\%)$ & QCD: & $2.3(2)$ & $2.0(2)$ \\
$\left(M_{t \bar{t}}<450 \mathrm{GeV}\right)$ & QCD + EW: & $2.7(2)$ & $2.3(2)$ \\
\hline
\end{tabular}

Table 13: The leptonic charge asymmetries (17) and (18) for dileptonic final states at the Tevatron, computed inclusively. The numbers in the third column were obtained by imposing the acceptance cuts (13). The uncertainties are due to scale variations $m_{t} / 2 \leq \mu \leq 2 m_{t}$. The results for $A^{\ell}$ without cuts apply also to lepton + jets final states (15).

\begin{tabular}{|c|c|c|c|}
\hline & $A^{\ell}(\%)$ & $A^{\ell}\left(M_{t \bar{t}}<450 \mathrm{GeV}\right)(\%)$ & $A^{\ell}\left(M_{t \bar{t}} \geq 450 \mathrm{GeV}\right)(\%)$ \\
\hline $\mathrm{D} \emptyset[2]$ & $15.2 \pm 4.0$ & & \\
\hline $\mathrm{CDF}[7]$ & $6.6 \pm 2.5$ & $3.7 \pm 3.1$ & $11.6 \pm 4.2$ \\
\hline
\end{tabular}

Table 14: The $\mathrm{D} \emptyset[2]$ and CDF [7] results for the leptonic charge asymmetry from $\ell+$ jet events at the Tevatron. The $\mathrm{D} \emptyset$ result is unfolded, while the CDF data are backgroundsubtracted results which are not yet corrected for detector effects and acceptance.

These asymmetries were first computed at NLO QCD (production and decay), including mixed QCD-weak (but not the QED) corrections, in 12]. The results of Table 13 are in agreement with these result $\$$. In [12] also the effect of $t \bar{t}$ spin correlations on the leptonic asymmetries was investigated. Switching the spin correlations off has only a minor effect, which is to be expected because the inclusive leptonic charge asymmetries are influenced but not primarily caused by $t, \bar{t}$ spin effects.

The asymmetry $A^{\ell}$ was also calculated in 25] for off-shell intermediate $t, \bar{t}$ at NLO QCD (production and decay, including non-factorizable corrections) and the result of [25] agrees with that of [12] and of Table 13. Recently another calculation of $A^{\ell}$ at NLO QCD (production and decay), $A^{\ell}=2.0_{-0.3}^{+1.0 \%}$, was reported in [35] for on-shell $t, \bar{t}$. As [35] uses the NLO QCD cross section in the denominator of $A^{\ell}$, which is $\sim 30 \%$ larger than $\sigma_{L O}$, this result is also in agreement with [12] and that of Table 13.

So far, the $\mathrm{D} \emptyset$ and $\mathrm{CDF}$ experiments have published only results for $A^{\ell}$ obtained from lepton plus jets final states, which we have collected in Table 14 for the convenience of the reader.

\footnotetext{
${ }^{8}$ In $[12$ the denominators of the asymmetries were evaluated with LO PDF.
} 
While the cited $\mathrm{D} \emptyset$ result is the unfolded one [2], the CDF results [7] are backgroundsubtracted but not yet corrected for detector effects and acceptance. According to [2], unfolding has only a minimal effect on the lepton asymmetry. Thus we may compare these experimental results with our SM predictions for $A^{\ell}$ in the no-cut case given in Table 13 which, as already mentioned above, apply also to $\ell+j$ events. While the CDF results agree with the SM predictions, the $\mathrm{D} \emptyset$ result $A_{\exp }^{\ell}=(15.2 \pm 4) \%$ deviates by $\sim 2.8 \sigma$.

\section{LHC}

At the LHC, leptonic charge asymmetries can be defined for dileptonic final states (12) in analogy to the $t \bar{t}$ charge asymmetries of Sect. 2.2. In analogy to (44) and (5) we define the leptonic center and edge asymmetries

$$
\begin{aligned}
& A_{C}^{\ell}\left(\eta_{c}\right)=\frac{N_{\ell \ell}\left(\left|\eta_{\ell^{+}}\right| \leq \eta_{c}\right)-N_{\ell \ell}\left(\left|\eta_{\ell^{-}}\right| \leq \eta_{c}\right)}{N_{\ell \ell}\left(\left|\eta_{\ell^{+}}\right| \leq \eta_{c}\right)+N_{\ell \ell}\left(\left|\eta_{\ell^{-}}\right| \leq \eta_{c}\right)}, \\
& A_{E}^{\ell}\left(\eta_{c}\right)=\frac{N_{\ell \ell}\left(\eta_{c} \leq\left|\eta_{\ell^{+}}\right|\right)-N_{\ell \ell}\left(\eta_{c} \leq\left|\eta_{\ell^{-}}\right|\right)}{N_{\ell \ell}\left(\eta_{c} \leq\left|\eta_{\ell^{+}}\right|\right)+N_{\ell \ell}\left(\eta_{c} \leq\left|\eta_{\ell^{-}}\right|\right)},
\end{aligned}
$$

where we choose in the following, for definiteness, $\eta_{c}=1$.

The cut-independent the $t \bar{t}$ asymmetry (9) translates to the asymmetry

$$
A^{\Delta\left|\eta_{\ell}\right|}=\frac{N_{\ell \ell}\left(\Delta\left|\eta_{\ell}\right|>0\right)-N_{\ell \ell}\left(\Delta\left|\eta_{\ell}\right|<0\right)}{N_{\ell \ell}\left(\Delta\left|\eta_{\ell}\right|>0\right)+N_{\ell \ell}\left(\Delta\left|\eta_{\ell}\right|<0\right)},
$$

where $\Delta\left|\eta_{\ell}\right|=\left|\eta_{\ell^{+}}\right|-\left|\eta_{\ell^{-}}\right|$.

Furthermore, we define

$$
\begin{aligned}
& A_{C}^{\Delta\left|\eta_{\ell}\right|}=A^{\Delta\left|\eta_{\ell}\right|} \quad \text { for events with }|\Delta| \eta_{\ell}|| \leq \eta_{c}, \\
& A_{E}^{\Delta\left|\eta_{\ell}\right|}=A^{\Delta\left|\eta_{\ell}\right|} \quad \text { for events with }|\Delta| \eta_{\ell}|| \geq \eta_{c},
\end{aligned}
$$

where we choose below $\eta_{c}=1$, too.

In Table 15 we collect our results for the leptonic charge asymmetries (19) - (23) for dileptonic final states for the LHC at 7, 8, and $14 \mathrm{TeV}$. The first set of numbers was computed using the acceptance cuts (14) and the anti- $k_{T}$ algorithm [50] with $R=0.5$. The second set of numbers was obtained by an inclusive calculation without imposing cuts. Our SM results for the leptonic asymmetries follow essentially the same pattern that was found in Sect. 2.2

for the corresponding charge asymmetries at the level of $t \bar{t}: A_{C}^{\Delta\left|\eta_{l}\right|}$ is negative and smaller in magnitude than $A_{E}^{\ell}$ and $A^{\Delta\left|\eta_{\ell}\right|}$. The edge or forward asymmetry may be enhanced by the additional selection cut (23). The $\eta_{\ell^{-}}$cut independent asymmetry $A^{\Delta\left|\eta_{\ell}\right|}$ can be enhanced by selecting events with high pair-invariant mass $M_{t \bar{t}}$, as shown in Table 16. For large $M_{t \bar{t}}$ the ratio of weak and QCD contributions increases somewhat.

So far, ATLAS and CMS have not yet published results on leptonic asymmetries from $t \bar{t}$ events. It will certainly be a challenge (that probably cannot be met) to detect nonzero effects being so small in magnitude than those given in Tables 15, 16. But the point is that these leptonic asymmetries should be excellent discriminators between SM and possible new physics effects, because it is expected that these asymmetries will be measurable with a precision of a few percent. 


\begin{tabular}{|c|c|c|c|c|c|c|}
\hline$\sqrt{s}$ & & $A_{C}^{\ell}$ & $A_{E}^{\ell}$ & $A^{\Delta\left|\eta_{\ell}\right|}$ & $A_{C}^{\Delta\left|\eta_{\ell}\right|}$ & $A_{E}^{\Delta\left|\eta_{\ell}\right|}$ \\
\hline \multicolumn{7}{|c|}{ With acceptance cuts (14) $\left(\right.$ anti- $k_{T}$ and $\left.R=0.5\right)$} \\
\hline $7 \mathrm{TeV}$ & QCD (\%): & $-0.25(1)$ & $0.41(2)$ & $0.41(2)$ & $0.23(1)$ & $0.95(4)$ \\
& QCD + EW (\%): & $-0.30(1)$ & $0.50(1)$ & $0.49(1)$ & $0.27(1)$ & $1.15(2)$ \\
\hline $8 \mathrm{TeV}$ & QCD (\%): & $-0.22(1)$ & $0.36(2)$ & $0.34(2)$ & $0.19(1)$ & $0.81(3)$ \\
& QCD + EW (\%): & $-0.27(1)$ & $0.43(1)$ & $0.42(1)$ & $0.23(1)$ & $0.98(2)$ \\
\hline $14 \mathrm{TeV}$ & QCD (\%): & $-0.09(1)$ & $0.14(1)$ & $0.09(1)$ & $0.03(1)$ & $0.25(2)$ \\
& QCD + EW (\%): & $-0.13(1)$ & $0.19(1)$ & $0.14(1)$ & $0.05(2)$ & $0.37(2)$ \\
\hline \multicolumn{7}{|c|}{ Without acceptance cuts } \\
\hline $7 \mathrm{TeV}$ & QCD (\%): & $-0.40(2)$ & $0.48(2)$ & $0.61(3)$ & $0.27(2)$ & $1.25(6)$ \\
& QCD + EW (\%): & $-0.46(2)$ & $0.55(2)$ & $0.70(3)$ & $0.32(2)$ & $1.44(6)$ \\
\hline $8 \mathrm{TeV}$ & QCD (\%): & $-0.36(2)$ & $0.42(2)$ & $0.55(3)$ & $0.25(1)$ & $1.13(6)$ \\
& QCD + EW (\%): & $-0.42(2)$ & $0.49(2)$ & $0.64(3)$ & $0.29(1)$ & $1.31(4)$ \\
\hline $14 \mathrm{TeV}$ & QCD (\%): & $-0.21(1)$ & $0.20(1)$ & $0.36(2)$ & $0.15(1)$ & $0.71(4)$ \\
& QCD + EW (\%): & $-0.25(1)$ & $0.24(1)$ & $0.43(2)$ & $0.17(1)$ & $0.85(3)$ \\
\hline
\end{tabular}

Table 15: The leptonic charge asymmetries for dileptonic final states for the LHC at 7,8 , and $14 \mathrm{TeV}$. The first set of numbers was computed using the acceptance cuts (14) and the anti- $k_{T}$ algorithm with $R=0.5$. The second set of numbers was obtained by an inclusive calculation without imposing cuts. The uncertainties are due to scale variations $m_{t} / 2 \leq \mu \leq 2 m_{t}$.

\begin{tabular}{|c|c|c|c|c|}
\hline$\sqrt{s}$ & $A^{\Delta\left|\eta_{l}\right|}(\%)$ & $M_{t \bar{t}} \geq 0.5 \mathrm{TeV}$ & $M_{t \bar{t}} \geq 0.7 \mathrm{TeV}$ & $M_{t \bar{t}} \geq 1 \mathrm{TeV}$ \\
\hline $7 \mathrm{TeV}$ & QCD: & $0.94(4)$ & $1.29(3)$ & $1.63(2)$ \\
& QCD+EW: & $1.13(2)$ & $1.53(2)$ & $1.94(1)$ \\
\hline $8 \mathrm{TeV}$ & QCD: & $0.85(3)$ & $1.16(3)$ & $1.43(9)$ \\
& QCD+EW: & $1.03(2)$ & $1.41(1)$ & $1.74(5)$ \\
\hline $14 \mathrm{TeV}$ & QCD: & $0.52(2)$ & $0.68(4)$ & $0.89(6)$ \\
& QCD+EW: & $0.67(2)$ & $0.88(2)$ & $1.13(3)$ \\
\hline
\end{tabular}

Table 16: The leptonic charge asymmetry $A^{\Delta\left|\eta_{l}\right|}$, for different cuts on $M_{t \bar{t}}$, for dileptonic final states at the LHC without acceptance cuts.

\section{Conclusions}

We have computed several top-quark charge asymmetries for $t \bar{t}$ production, for the Tevatron and in particular for the LHC, to NLO QCD including mixed QCD-QED and QCD-weak interaction corrections. Our SM prediction for the $t \bar{t}$ rest-frame asymmetry at high mass, $A^{t \bar{t}}\left(M_{t \bar{t}}>450 \mathrm{GeV}\right)$ (and those by other authors [11, 16]) at the Tevatron deviates from the recent CDF measurement [7] by $\sim 2.4 \sigma$. Thus, for this observable, the tension between experiment and SM has become less severe, as compared to the situation about 1 year ago [1]. For the LHC we have made SM predictions for a number of top charge asymmetries that were proposed in the literature, namely for the center and edge asymmetry, the rapidity-cut independent asymmetries (without and with additional cuts on $M_{t \bar{t}}$ ), and for the boosted and one-sided asymmetry. The measurements of the inclusive asymmetries $A_{C}^{\Delta|\eta|}, A_{C}^{\Delta|y|}$ by the CMS and ATLAS experiments agree with the SM results; the predictions for $t \bar{t}$ samples with 
large pair-invariant mass and for the other charge asymmetries still need to be experimentally tested.

Moreover, we have considered several leptonic charge asymmetries for dileptonic and lepton + jets $t \bar{t}$ events at the Tevatron and for dileptonic $t \bar{t}$ events at the LHC. We have computed these asymmetries at NLO QCD (production and decay), including the mixed QCD-electroweak corrections to $t \bar{t}$ production. These leptonic asymmetries should be measurable rather precisely and provide additional information about $t \bar{t}$ production. While the CDF results on $A^{\ell}$ (not yet unfolded) agree with our predictions, the unfolded $\mathrm{D} \emptyset$ result deviates by $\sim 2.8 \sigma$. Hopefully, $\mathrm{D} \emptyset$ and $\mathrm{CDF}$ will perform also measurements of the asymmetry $A^{\ell \ell}$ for dileptonic events. In addition, we expect that results on the LHC leptonic charge asymmetries, for which we presented SM predictions in Tables 15 and 16, will become available from ATLAS and/or CMS in the not too distant future. The measurements of these and other distributions, including the search for an non-zero longitudinal polarization of the top (anti)quarks in hadronically produced $t \bar{t}$ samples and measurements of $t \bar{t}$ spin correlations with increased precision 9 should eventually clarify in detail the dynamics of hadronic $t \bar{t}$ production and decay.

\section{Acknowledgements}

We wish to thank D. Amidei, A. Harel, B. Pecjak, and C. Schwanenberger for discussions and correspondence. The work of W.B. was supported by DFG, SFB TR9 and that of Z.G. Si by NSFC and by Natural Science Foundation of Shandong Province.

\section{Note added}

After submission of this manuscript several new experimental results appeared that are of relevance for some of our results. The $\mathrm{D} \emptyset$ collaboration at the Tevatron reported the measurement of the leptonic asymmetries (16) - (18) for dilepton final states and obtained the unfolded results [55]: $A^{\ell}=(5.8 \pm 5.1$ (stat) $\pm 1.3($ syst $)) \%$ and $A^{\ell \ell}=(5.3 \pm 7.9$ (stat) $\pm 2.9($ syst $)) \%$, which are in agreement with our results given in Table 13. The ATLAS collaboration reported the measurement of the leptonic asymmetry (21) for dilepton final states at the LHC $(7 \mathrm{TeV})[56], A^{\Delta\left|\eta_{\ell}\right|}=(2.3 \pm 1.2$ (stat) \pm 0.8 (syst) $) \%$. This measurement is in agreement with our corresponing result (without acceptance cuts) given in Table 15. Moreover, the recent measurements of the $t \bar{t}$ charge asymmetry $A_{C}^{\Delta|y|}$ by the ATLAS [56] and CMS [57] experiments at the $\mathrm{LHC}(7 \mathrm{TeV})$ are compatible with our SM prediction given in Table 8 .

\footnotetext{
${ }^{9}$ The size of the SM weak-interaction induced longitudinal polarization of the top (anti)quarks was determined in 12,41,42. Evidence and observation of non-zero $t \bar{t}$ spin correlations was recently reported by $\mathrm{D} \emptyset[52$ and by ATLAS [53, respectively.
} 


\section{References}

[1] T. Aaltonen et al. [CDF Collaboration], Phys. Rev. D 83, 112003 (2011) arXiv:1101.0034 [hep-ex]].

[2] V. M. Abazov et al. [D0 Collaboration], Phys. Rev. D 84, 112005 (2011) arXiv:1107.4995 [hep-ex]].

[3] J. H. Kühn and G. Rodrigo, Phys. Rev. Lett. 81, 49 (1998) hep-ph/9802268.

[4] J. H. Kühn and G. Rodrigo, Phys. Rev. D 59, 054017 (1999) arXiv:hep-ph/9807420|.

[5] M. T. Bowen, S. D. Ellis and D. Rainwater, Phys. Rev. D 73, 014008 (2006) arXiv:hep-ph/0509267.

[6] O. Antunano, J. H. Kühn and G. V. Rodrigo, Phys. Rev. D 77, 014003 (2008) arXiv:0709.1652 [hep-ph]].

[7] T. Aaltonen et al. [CDF Collaboration], CDF note 10807.

[8] J. F. Kamenik, J. Shu and J. Zupan, arXiv:1107.5257 [hep-ph].

[9] S. Westhoff, arXiv:1108.3341 [hep-ph].

[10] J. A. Aguilar-Saavedra, arXiv:1202.2382 [hep-ph].

[11] W. Hollik and D. Pagani, Phys. Rev. D 84, 093003 (2011) [arXiv:1107.2606 [hep-ph]].

[12] W. Bernreuther and Z. -G. Si, Nucl. Phys. B 837, 90 (2010) [arXiv:1003.3926 [hep-ph]].

[13] S. Chatrchyan et al. [CMS Collaboration], Phys. Lett. B 709, 28 (2012) arXiv:1112.5100 [hep-ex]].

[14] [CMS Collaboration], report CMS-PAS-TOP-11-030 (2012).

[15] G. Aad et al. [ATLAS Collaboration], arXiv:1203.4211 [hep-ex].

[16] J. H. Kühn and G. Rodrigo, JHEP 1201, 063 (2012) [arXiv:1109.6830 [hep-ph]].

[17] Y. -k. Wang, B. Xiao and S. -h. Zhu, Phys. Rev. D 82, 094011 (2010) arXiv:1008.2685 [hep-ph]].

[18] B. Xiao, Y. -K. Wang, Z. -Q. Zhou and S. -h. Zhu, Phys. Rev. D 83, 057503 (2011) arXiv:1101.2507 [hep-ph]].

[19] J. L. Hewett, J. Shelton, M. Spannowsky, T. M. P. Tait and M. Takeuchi, Phys. Rev. D 84, 054005 (2011) arXiv:1103.4618 [hep-ph]].

[20] J. -F. Arguin, M. Freytsis and Z. Ligeti, Phys. Rev. D 84, 071504 (2011) arXiv:1107.4090 [hep-ph]].

[21] J. A. Aguilar-Saavedra, A. Juste and F. Rubbo, Phys. Lett. B 707, 92 (2012) arXiv:1109.3710 [hep-ph]]. 
[22] E. Alvarez, arXiv:1202.6622 [hep-ph].

[23] E. Alvarez, arXiv:1205.5267 [hep-ph].

[24] J. A. Aguilar-Saavedra and A. Juste, arXiv:1205.1898 [hep-ph].

[25] G. Bevilacqua, M. Czakon, A. van Hameren, C. G. Papadopoulos and M. Worek, JHEP 1102, 083 (2011) [arXiv:1012.4230 [hep-ph]].

[26] S. Dittmaier, P. Uwer and S. Weinzierl, Phys. Rev. Lett. 98, 262002 (2007) [arXiv:hep-ph/0703120].

[27] S. Dittmaier, P. Uwer and S. Weinzierl, Eur. Phys. J. C 59, 625 (2009) arXiv:0810.0452 [hep-ph]].

[28] K. Melnikov and M. Schulze, Nucl. Phys. B 840, 129 (2010) [arXiv:1004.3284 [hep-ph]].

[29] K. Melnikov, A. Scharf and M. Schulze, Phys. Rev. D 85, 054002 (2012) arXiv:1111.4991 [hep-ph]].

[30] S. Alioli, S. -O. Moch and P. Uwer, JHEP 1201, 137 (2012) [arXiv:1110.5251 [hep-ph]].

[31] L. G. Almeida, G. Sterman and W. Vogelsang, Phys. Rev. D 78, 014008 (2008) arXiv:0805.1885 [hep-ph]].

[32] V. Ahrens, A. Ferroglia, M. Neubert, B. D. Pecjak and L. L. Yang, JHEP 1009, 097 (2010) arXiv:1003.5827 [hep-ph]].

[33] N. Kidonakis, Phys. Rev. D 84, 011504 (2011) [arXiv:1105.5167 [hep-ph]].

[34] V. Ahrens, A. Ferroglia, M. Neubert, B. D. Pecjak and L. L. Yang, Phys. Rev. D 84, 074004 (2011) arXiv:1106.6051 [hep-ph]].

[35] J. M. Campbell and R. K. Ellis, arXiv:1204.1513 [hep-ph].

[36] S. Frixione and B. R. Webber, arXiv:0812.0770 [hep-ph].

[37] S. Frixione, P. Nason and G. Ridolfi, JHEP 0709, 126 (2007) [arXiv:0707.3088 [hep-ph]].

[38] J. Campbell and R. K. Ellis, http:/mcfm.fnal.gov

[39] J. M. Campbell and R. K. Ellis, Nucl. Phys. Proc. Suppl. 205-206, 10 (2010) arXiv:1007.3492 [hep-ph]].

[40] W. Bernreuther, M. Fuecker and Z. G. Si, Phys. Lett. B 633, 54 (2006) arXiv:hep-ph/0508091].

[41] W. Bernreuther, M. Fuecker and Z. G. Si, Phys. Rev. D 74, 113005 (2006) arXiv:hep-ph/0610334.

[42] W. Bernreuther, M. Fücker and Z. G. Si, Phys. Rev. D 78, 017503 (2008) arXiv:0804.1237 [hep-ph]]. 
[43] J. H. Kühn, A. Scharf and P. Uwer, Eur. Phys. J. C 45, 139 (2006) arXiv:hep-ph/0508092.

[44] J. H. Kühn, A. Scharf and P. Uwer, Eur. Phys. J. C 51, 37 (2007) arXiv:hep-ph/0610335.

[45] W. Beenakker, A. Denner, W. Hollik, R. Mertig, T. Sack and D. Wackeroth, Nucl. Phys. B 411, 343 (1994).

[46] S. J. Brodsky and X. -G. Wu, arXiv:1205.1232 [hep-ph].

[47] P. M. Nadolsky, H. -L. Lai, Q. -H. Cao, J. Huston, J. Pumplin, D. Stump, W. -K. Tung and C. -P. Yuan, Phys. Rev. D 78, 013004 (2008) [arXiv:0802.0007 [hep-ph]].

[48] A. D. Martin, W. J. Stirling, R. S. Thorne and G. Watt, Eur. Phys. J. C 63, 189 (2009) arXiv:0901.0002 [hep-ph]].

[49] S. Catani, Y. L. Dokshitzer and B. R. Webber, Phys. Lett. B 285, 291 (1992).

[50] M. Cacciari, G. P. Salam and G. Soyez, JHEP 0804, 063 (2008) arXiv:0802.1189 [hep$\mathrm{ph}]$.

[51] A. Falkowski, G. Perez and M. Schmaltz, arXiv:1110.3796 [hep-ph].

[52] V. M. Abazov et al. [D0 Collaboration], Phys. Rev. Lett. 108, 032004 (2012) arXiv:1110.4194 [hep-ex]].

[53] G. Aad et al. [ATLAS Collaboration], arXiv:1203.4081 [hep-ex].

[54] J. Drobnak, J. F. Kamenik and J. Zupan, arXiv:1205.4721 [hep-ph].

[55] V. M. Abazov et al. [D0 Collaboration], arXiv:1207.0364 [hep-ex].

[56] G. Aad et al. [ATLAS Collaboration], report ATLAS-CONF-2012-057.

[57] S. Chatrchyan et al. [CMS Collaboration], arXiv:1207.0065 [hep-ex]. 